\title{
ON THE MEASURE OF TOTALLY REAL ALGEBRAIC INTEGERS
}

\author{
C. J. SMYTH \\ (Received 4 October 1979) \\ Communicated by A. J. van der Poorten
}

\begin{abstract}
For totally real algebraic integer $x$ of degree $D(x)$, we examine the stucture of the set of values $M(x)^{1 D(x)}$, where $M(x)$ is the measure of $x$. We find a small limit point $f$ of this set, and show that the set is everywhere dense in $(f, x)$.
\end{abstract}

1980). Wathematics subject classification (Amer. Math. Soc.): 12 A 95.

\section{Introduction}

Let $x \neq 0$ be an algebraic integer, not a root of unity, with conjugates $x=x_{1}, x_{2}, \ldots, x_{D(x)}$. There has been much recent work on the product $M(x)=\prod_{i=1}^{D(x)} \max \left(1,\left|\alpha_{i}\right|\right)$ (see Boyd (1978), Mignotte (1978), Stewart (1978) and forthcoming papers of Dobrowolski, Lawton and Schinzel).

Here we shall be concerned with $M(x)$ for $\alpha$ a totally real algebraic integer, $\alpha \neq 0$, \pm 1 . In this situation, a reformulation of a special case of a result of Schinzel (1973), Theorem 2, states that

$$
M(x) \geqslant\left(\frac{1+\sqrt{5}}{2}\right)^{\frac{1}{2} D(x)}
$$

with equality when $x=\frac{1}{2}(1+\sqrt{ } 5)$. It therefore seems reasonable to put $\Omega(x)=M(x)^{1 D(x)}$ and look at the set

$$
\mathscr{L}=\{\Omega(x) \mid x \text { totally real, } x \neq 0, \pm 1\} .
$$

Then by Schinzel's result, $\mathscr{L}$ has smallest element $\left(\frac{1}{2}(1+\sqrt{ } 5)\right)^{\frac{2}{2}}=1.2720196 \ldots$. We shall prove the following results:

THeOREM 1. Define $\beta_{0}=1$ and $\beta_{n+1}>0$ by $H \beta_{n+1}=\beta_{n}(n=0,1, \ldots)$, where 


$$
H x=x-x^{-1}
$$

Then $\beta_{n}$ has degree $2^{n}$ over the rationals, and the sequence

$$
\Omega\left(\beta_{1}\right), \Omega\left(\beta_{2}\right), \Omega\left(\beta_{3}\right), \Omega\left(\beta_{4}\right), \Omega\left(\beta_{5}\right), \ldots \approx 1.272,1.298,1.308,1.312,1.313, \ldots
$$

of elements of $\mathscr{L}$ has limit point

$$
\ell=\exp \int_{1}^{x} \log x d F(x)=1.31427 \ldots
$$

where $F(x)$ is the function defined by Theorem 3.

THEOREM 2. The set $\mathscr{L}$ is everywhere dense in the intercal $(\ell, x)$.

THEOREM 3. There is a unique strictly increasing function $F(x)$, defined on $[0, x]$ and satisfying $F(0)=0$ and

$$
|2 F(x)-1|=F\left(\left|x-x^{-1}\right|\right)(x \geqslant 0) .
$$

The function $F(x)$ is in fact the limiting distribution, as $n \rightarrow x$, of the absolute talues of the conjugates of $\beta_{n}$.

It would be interesting to determine the precise structure of $\mathscr{L}$ in $\left(\left(\frac{1}{2}(1+\sqrt{5})\right)^{\frac{1}{2}} . \ell\right.$. It seems likely that the $\Omega\left(\beta_{n}\right)(n=1,2, \ldots)$ form an increasing sequence lying entirely within this interval, though I have not been able to prove this. Apart from the $\Omega\left(\beta_{n}\right)$. there are other elements of $\mathscr{L}$ in this interval. They are connected with fixed points of iterates $H^{k}$ of $H$. These are discussed in Section 6.

One might expect that the numbers $\Omega\left(x_{q}\right)$. where

$$
\alpha_{q}=2 \cos (2 \pi / q)
$$

could give small elements of $\mathscr{L}$. In fact, $\lim _{q \rightarrow \gamma} \Omega\left(x_{q}\right)=1.38135 \ldots>\ell$ (Lemma 11). and I know of no $\Omega\left(x_{q}\right)$ on $\left(\left(\frac{1}{2}(1+\sqrt{5})\right)^{\frac{1}{2}}, \ell\right)$ which is not also equal to $\Omega\left(\beta_{n}\right)$ for some $n$, or $\Omega\left(\beta^{\prime}\right)$ for some fixed point $\beta^{\prime}$ of $H^{k}$ for some $k$ (see Section 6 for details).

In Section 2 we calculate the degree of $\beta_{n}$. In Section 3 we prove Theorem 3, and derive some other properties of $\beta_{n}$ and $F$. In Section 4 we complete the proof of Theorem 1, and in Section 5 we prove Theorem 2.

I would like to thank Professor J. W. S. Cassels for useful discussions concerning the degree of $\beta_{n}$. 


\section{Degree of $\beta_{n}$}

LEMMA 1 (Albert (1956). Theorem 22, p. 140). Let $p$ be a prime and $\gamma \in \mathrm{GF}\left(p^{n}\right)$ for some $n$. Then $x^{p}-x-;$ is irreducible over $\mathrm{GF}\left(p^{n}\right)$ if and only if the trace $\operatorname{Tr}_{\text {(if Im) Gitim) }} \hat{\prime} \neq 0$.

LEMMA 2. If $x \neq 0$ belongs to a field of characteristic 2 , and $\mu=x^{-1}+x$ satisfies $\mu^{2{ }^{n}}=\mu$ for some $n$, then $x^{2 \uparrow n}=x$ or $x^{-1}$. Here $2 \uparrow n$ denotes $2^{2 n}$.

PROOF. Now $\left(x+x^{-1}\right)^{2{ }^{n}}=x^{2 \uparrow n}+x^{-2 \uparrow n}=\mu^{2 \dagger n}=\mu$. So $x^{2 \uparrow n}$ is one of the roots of $x+x^{-1}=\mu$.

LEMMA 3. In a suitable extenstion of $F_{2}=\mathrm{GF}(2)$, define $\gamma_{0}=1$ and

$$
i_{n+1}+i_{n+1}^{-1}=\ddot{i n}_{n} \quad(n=0,1,2, \ldots) .
$$

Then $\left[F_{2}\left(i_{i n}\right): F_{2}\right]=2 n$.

PROOF. Assume for inductive purposes that $\left[F_{2}\left(\gamma_{n}\right): F_{2}\right]=2^{n}, \operatorname{Tr}_{F_{2}(; n) F_{2} \gamma_{n}}=1$ and $i_{n}^{2}{ }^{*}(n-1)=i_{n}^{-1}$. This is easily verified for $n=1$. Then $\left(\gamma_{n+1} / \gamma_{n}\right)^{2}+\gamma_{n+1} / \gamma_{n}=\gamma_{n}^{-2}$, and $\operatorname{Tr}_{i n}^{-2}=\operatorname{Tr}{ }_{i n}$ as $i_{i n}^{-2}=i_{n}^{2(2 \uparrow(n-1)}$ and $\operatorname{Tr} \gamma_{n}=\sum_{j=1}^{2^{n}-1} i_{n}^{2}$. So by Lemma 1 , $i_{n+1} / i_{n} \notin F_{2}\left(i_{n}\right)$, and hence $\left[F_{2}\left(\gamma_{n+1}\right): F_{2}\right]=2^{n+1}$. Since $\gamma_{n+1} \notin F_{2}\left(\gamma_{n}\right), \gamma_{n+1}^{2+n} \neq \gamma_{n+1}$, so $i_{n+1}^{2}=i_{n+1}^{-1}$ by Lemma 2. Further, from (2.1)

$$
i_{n+1}^{2 k}+i_{n+1}^{-2 k}=\gamma_{n}^{2 k} \quad\left(k=0,1, \ldots, 2^{n}-1\right) .
$$

Since $(i n+1)^{2}=i_{n+1}^{2} n=i n+1$, where $e=2^{n}-1$, it follows that

$$
\operatorname{Tr}_{n-1}^{n}=\sum_{k=1}^{2^{n}-1}\left(\gamma_{n+1}^{2^{k}}+\gamma_{n+1}^{-2^{k}}\right)=\sum_{k=1}^{2^{n}-1} \gamma_{n}^{2^{k}}=1 \text {, }
$$

by the induction hypothesis. This completes the induction.

We can now prove

Lemma 4. Let $\beta_{0}$ be an odd rational integer, and $H \beta_{n+1}=\beta_{n}(n=0,1, \ldots)$. Then $\beta_{n}$ has degree $2^{n}$ over the rationals $Q$.

ProOF. We show that $Q_{2}\left(\beta_{n}\right) / Q_{2}$ is unramified of degree $2^{n}$, where $Q_{2}$ is the field of 2-adic numbers. Assume inductively that $Q_{2}\left(\beta_{n}\right)$ has residue class field $F_{2}\left(i_{i}\right)$, and that $\beta_{n} \equiv i_{n}(\bmod 2)$ (clearly true for $\left.n=0\right)$. Then if $f(x)=x^{2}-\beta_{n} x+1$, $f\left(i_{n+1}\right) \equiv 0(\bmod 2)$, and $f^{\prime}\left(\gamma_{n+1}\right) \equiv \hat{\gamma}_{n} \hat{\gamma}_{n+1} \not \equiv 0(\bmod 2)$. So by Hensel's Lemma, $f(x)=0$ has a $\operatorname{root} \beta_{n+1}$ with $\beta_{n+1} \equiv \gamma_{n+1}(\bmod 2)$. Then $Q_{2}\left(\beta_{n+1}\right)$ has residue class field $F_{2}\left(i_{n}, i_{n+1}\right)=F_{2}\left(\hat{i}_{n+1}\right)$ of degree $2^{n+1}$ over $F_{2}$, by Lemma 3 . Hence 


$$
\left[Q_{2}\left(\beta_{n+1}\right): Q_{2}\right] \geqslant\left[F_{2}\left(i_{n+1}\right): F_{2}\right]=2^{n+1},
$$

and $Q_{2}\left(\beta_{n+1}\right) / Q_{2}$ is unramified of degree $2^{n+1}$.

\section{Proof of Theorem 3}

Let $B_{n}$ be the set of absolute values of conjugates of $\beta_{n}(n=0,1, \ldots)$. By Lemma 4 . $B_{n}$ has $2^{n}$ elements $\beta_{n}=\beta_{n, 1} \geqslant \beta_{n, 2} \geqslant \ldots \geqslant \beta_{n, 2^{n}}$ say. For $x \geqslant 0$, put $F_{n}(x)=2^{-n} \times\left(\right.$ number of $\beta_{n, j}$ in $\left.[0, x]\right)$. Clearly $F_{n}(0)=0$. Since $-\beta_{n}^{-1}$ is a conjugate of $\beta_{n}$,

$$
F_{n}(x)= \begin{cases}1-F_{n}\left(x^{-1}\right) & \text { if } x \neq a n y \beta_{n, j}, \\ 1-F_{n}\left(x^{-1}\right)+2^{-n} & \text { if } x=\operatorname{som} c \beta_{n, j}\end{cases}
$$

Also, for $x>1$ there is a 11 correspondence between the $\beta_{n, j}$ in $\left(x, x_{-}\right)$and the $\beta_{n-1, j}$ in $\left(x-x^{-1}, x\right)$. So $2^{n}\left(1-F_{n}(x)\right)=2^{n-1}\left(1-F_{n-1}\left(x-x^{-1}\right)\right)$. or

$$
F_{n}(x)=\frac{1}{2}\left(1+F_{n-1}\left(x-x^{-1}\right)\right), \quad x>1 .
$$

Now take any $x \geqslant 0$. If $x \in \bigcup_{j=0}^{n} B_{j}$, replace $x$ by $x^{\prime}>x: F_{n}\left(x^{\prime}\right)=F_{n}(x)$. $F_{n-1}\left(x^{\prime}\right)=F_{n-i}(x)$ and $x^{\prime} \notin \bigcup_{j=0}^{n} B_{j}$. So we can assume in what follows that $x \notin \bigcup_{j=0}^{n} B_{j}$, which implies by (3.1) that

$$
F_{j}(x)+F_{j}\left(x^{-1}\right)=1 \quad(j=0, \ldots, n) .
$$

From (3.2) and (3.3), for $y \notin B_{j}, B_{j-1}, y>0$,

$$
\left|F_{j}(y)-F_{j-1}(y)\right|=\frac{1}{2}\left|F_{j-1}\left(\left|y-y^{-1}\right|\right)-F_{j-2}\left(\left|y-y^{-1}\right|\right)\right| .
$$

Since $y \notin \bigcup_{j=0}^{n} B_{j}$ implies $\left|y-y^{-1}\right| \notin \bigcup_{j=0}^{n} B_{j}$, we have by induction that

$$
\left|F_{n}(x)-F_{n-1}(x)\right|=2^{-(n-2)}\left|F_{2}(z)-F_{1}(z)\right| \leqslant 2^{-(n) 2)} \text { for some } z
$$

By the Weierstrass $M$-test. $F_{n}(x)$ tends uniformly in $x$ to a limit function $F(x)$ say, as $|F(x)-F(x+\delta)| \leqslant 2^{-k}<\varepsilon$, from which continuity follows.

$$
\begin{gathered}
F(x)+F\left(x^{-1}\right)=1 \\
F(x)=\frac{1}{2}\left(1+F\left(x-x^{-1}\right)\right), \quad x \geqslant 1
\end{gathered}
$$

and hence

$$
F(x)=\frac{1}{2}\left(1-F\left(x^{-1}-x\right)\right)=\frac{1}{2} F\left(\left(x^{-1}-x\right)^{-1}\right) \quad(0 \leqslant x \leqslant 1) .
$$

Combining (3.5), (3.6) we can write them as (1.2). Conversely, under the assumption that $F$ is strictly increasing, (1.2) easily implies (3.4), (3.5) and (3.6). We shall show in Lemma 8 that $F$ is indeed strictly increasing. 
We now show how to use (3.5) and (3.6) to obtain, for given $x$, the value of $F(x)$ to any specified degree of accuracy. Suppose we have obtained an equation of the form

$$
F(x)=a_{k}+\varepsilon_{k} 2^{-k} F\left(\left|H^{k} x\right|\right),
$$

where $a_{k}$ is a rational, $\varepsilon_{k}= \pm 1$ and $H^{k} x=H\left(H^{k-1} x\right)$. (We start with $k=0, a_{0}=0$, $\varepsilon_{0}=1, H^{0} x=x$.) Then, applying (3.5) or (3.6),

$$
\begin{aligned}
F(x) & =a_{k}+\varepsilon_{k} 2^{-k}\left(\frac{1}{2}+\frac{1}{2} \varepsilon_{k+1}^{\prime} F\left(\left|H^{k+1} x\right|\right)\right. \\
& =a_{k+1}+\varepsilon_{k+1} 2^{-(k+1)} F\left(\left|H^{k+1} x\right|\right) \text { say. }
\end{aligned}
$$

So we can get an equation of the form (3.7) for any $k$, and then $\left|F(x)-a_{k}\right| \leqslant 2^{-k}$. This shows also that $F$ is uniquely defined by (3.5) and (3.6).

For later use, we need the following facts :

Lemma 5. (a) Define $H^{-1} x=\frac{1}{2}\left(x+\left(x^{2}+4\right)^{\frac{1}{2}}\right)$, so that $H\left(H^{-1} x\right)=x$ (and also $\left.H\left(\left(-H^{-1} x\right)^{-1}\right)=x\right)$. Then for $x, y>0$

$$
\left|H^{-1} x-H^{-1} y\right|<|x-y| \text {. }
$$

(b) We hate

$$
B_{n-1}=H^{-1} B_{n} \cup\left(H^{-1} B_{n}\right)^{-1}
$$

and for $n \geqslant 0$,

$$
\beta_{n+1, i}=H^{* 1} \beta_{n, i} \quad, \beta_{n+1, i^{i}}=\left(H^{-1} \beta_{n, i}\right)^{-1} \quad\left(i=1, \ldots, 2^{n}\right)
$$

where $i^{\prime}=2^{n+1}+1-i$.

(c) $(n+1)^{\frac{1}{2}} \leqslant \beta_{n} \leqslant(2 n+1)^{\frac{1}{2}}$ for $n \geqslant 0$.

(d) $\beta_{n}-\beta_{n, 2} \geqslant \beta_{n-1}^{-1}$ for $n \geqslant 1$ (recall $\beta_{n}, \beta_{n, 2}$ are the largest two elements of $B_{n}$ ).

(e) $\max _{j=1 \ldots \ldots 2 n-1}\left(\beta_{n, j}-\beta_{n, j+1}\right)=\beta_{n}-\beta_{n, 2}$, which is $O\left(n^{-\frac{1}{2}}\right)$.

Proof. (a) Direct application of the mean value theorem.

(b) (3.8) follows from (1.1), and (3.9) from (3.8).

(c) First note that $\beta_{n+1}=H^{-1} \beta_{n}=\frac{1}{2}\left(\beta_{n}+\left(\beta_{n}^{2}+4\right)^{\frac{1}{2}}\right)>\beta_{n}+\left(1 /\left(2 \beta_{n}\right)\right)$ as $\beta_{n}^{2}+4>\left(\beta_{n}+\beta_{n}^{-1}\right)^{2}$. Now assume $\beta_{n} \geqslant(n+1)^{\frac{1}{2}}$, which is true for $n=0$. Then

$$
\beta_{n-1}^{2}>\left((n+1)^{\frac{1}{2}}+\frac{1}{2}(n+1)^{\frac{1}{2}}\right)^{2}>n+2 .
$$

Next assume $\beta_{n} \leqslant(2 n+1)^{\frac{1}{2}}$, also true for $n=0$. Then

$$
\beta_{n+1} \leqslant \frac{1}{2}\left((2 n+1)^{\frac{1}{2}}+(2 n+5)^{\frac{1}{2}}\right) \leqslant(2 n+3)^{\frac{1}{2}}
$$

by convexity.

(d) We must first show that for $n \geqslant 1$

$$
\beta_{n}-\beta_{n-1} \geqslant \beta_{n, 2}-\beta_{n-2}
$$


(put $\beta_{-1}=0$ ). This holds with equality for $n=1$. Now, using (3.9),

$$
\begin{gathered}
\beta_{n}=H^{-(n-1)}\left(H^{-1} 1\right), \quad \beta_{n-1}=H^{-(n-1)} 1, \\
\beta_{n, 2}=H^{-(n-1)}\left(\left(H^{-1} 1\right)^{-1}\right), \quad \beta_{n-2}=H^{-(n-1)} 0 \text { and } H^{-1} 1>1>\left(H^{-1} 1\right)^{-1}>0
\end{gathered}
$$

Further, $(d / d x)\left(H^{-1} x\right)$ is an increasing function of $x$, so using the mean value theorem we have that if $a>b>c>d$ and $a-b>c-d$, then $H^{-1} a-H^{-1} b>H^{-1} c-H^{-1} d$. Applying this result $n-1$ times, (3.10) follows. Then (d) follows from the fact that $\beta_{n-2}=\beta_{n-1}-\beta_{n-1}^{-1}$.

(e) Now $\left|x^{-1}-y^{-1}\right|<|x-y|$ for $x, y-1$. So, using (3.8), the greatest distance between adjacent elements of $B_{n+1}$ must either occur between two elements of $H^{-1} B_{n}$, or between the smallest element $H^{-1}\left(\beta_{n}^{-1}\right)$ of $H^{-1} B_{n}$ and the largest element $\left(H^{-1}\left(\beta_{n}^{-1}\right)\right)^{-1}$ of $\left(H^{-1} B_{n}\right)^{-1}$. But

$$
H^{-1} \beta_{n}^{-1}-\left(H^{-1} \beta_{n}^{-1}\right)^{-1}=H H^{-1} \beta_{n}^{-1}=\beta_{n}^{-1} \leqslant \beta_{n+1}-\beta_{n+1,2}
$$

by (d), so if the result is true for $n$ it is also true for $n+1$. For the order of magnitude, first note that

$$
H \beta_{j}-H \beta_{j, 2}=\left(\beta_{j}-\beta_{j, 2}\right)\left(1+\frac{1}{x^{2}}\right) .
$$

for some $x \in\left(\beta_{j, 2}, \beta_{j}\right)$. Hence

$$
\beta_{j-1}-\beta_{j-1.2} \geqslant\left(\beta_{j}-\beta_{j, 2}\right)\left(1+\frac{1}{\beta_{j}^{2}}\right) \geqslant\left(\beta_{j}-\beta_{j, 2}\right)\left(\frac{2 j+2}{2 j+1}\right)
$$

by (c). Hence by induction, for $n \geqslant 2$

$$
\beta_{n}-\beta_{n, 2} \leqslant \frac{2 n+1}{2 n+2} \cdot \frac{2 n-1}{2 n} \cdot \cdots \cdot \frac{5}{6}
$$

as $\beta_{1}-\beta_{1,2}=1$. Since this product is $O\left(n^{-\frac{1}{2}}\right)$, the result follows.

LEMMA 6. $F$ is continuous on $(0, \infty)$.

Proof. Given $x, \varepsilon>0$, choose $k: 2^{-k}<\varepsilon$, and $\delta>0$ such that for $j=0,1, \ldots, k-1$. $\left|H^{j} x\right|$ and $\left|H^{j}(x+\delta)\right|$ are not on opposite sides of 1 (one of them may equal 1). This is possible by the continuity of $H$ on $R \cup\{x\}$ (with its usual topology). Then (3.7) holds for $x$ and $x+\delta$, with the same values of $a_{k}$ and $\varepsilon_{k}$. Hence $|F(x)-F(x+\delta)| \leqslant 2^{-k}<\varepsilon$ from which continuity follows.

LeMma 7. For $j=1, \ldots, 2^{n}, F\left(\beta_{n, j}\right)=1-(2 j-1) / 2^{n+1}$.

Proof. If $F(|\beta|)=j^{\prime} / 2^{n+1}$, where $j^{\prime}$ is odd, then repeated use of (1.2) shows that $H^{n}( \pm \beta)=1$. Hence by the definition of $\beta_{n}$, one of $\beta$ or $-\beta$ is a conjugate of $\beta_{n}$. Since 
$F(x)$ is continuous, $F(0)=0, F(x)=1, F(x)=j^{\prime} / 2^{n+1}$ has a solution $x_{j^{\prime}}$ say. So the $2^{n}$ odd values of $j^{\prime}$ in $\left[1,2^{n+1}-1\right]$ must correspond to the absolute values of the $2^{n}$ roots of $H^{n} x=1$. The exact correspondence follows from the ordering of the $\beta_{n, j}$ 's and the fact that $F$ is non-decreasing.

Finally in this section we can show

LeMma $8 . F$ is strictly increasing in $(0, \infty)$.

Proof. Let $0<a<b$. Choose $n$ large enough so that there are two elements $\beta_{n, j}$, $\beta_{n, j-1}$ of $B_{n}$ in $(a, b)$. This is possible by Lemma 5(c), (e). Then $F(a) \leqslant F\left(\beta_{n, j}\right)<F\left(\beta_{n, j-1}\right) \leqslant F(b)$.

The above result completes the proof of Theorem 2 .

\section{Proof of Theorem 1}

Lemma 9. We have

(a)

$$
\int_{1}^{x} \log x d F(x)=\log \ell
$$

for some $\ell$ with $1<\ell<x$.

(b)

$$
\lim _{n \rightarrow x} \int_{1}^{x} \log x d F_{n}(x)=\log \ell
$$

ProOF. (a)

$$
\begin{aligned}
\int_{1}^{x} \log x d F(x) & =\sum_{i=0}^{x} \int_{\beta_{i}}^{\beta_{i+1}} \log x d F(x) \\
& \leqslant \sum_{i=0}^{x} \log \beta_{i+1} \int_{\beta_{i}}^{\beta_{i}+1} d F(x) \\
& =\sum_{i=0}^{x} 2^{-(i+2)} \log \beta_{i+1} \leqslant \sum_{i=0}^{\infty} 2^{-(i+3)} \log (2 i+3)<x .
\end{aligned}
$$

by Lemma 7 and Lemma $5(\mathrm{c})$.

(b) Given $\varepsilon>0$, put $\varepsilon=2^{n}-1$ and choose $n$ :

$$
\left|\int_{1}^{\beta_{n, .}} \log x d F(x)-2^{-n} \log \beta_{n, e}\right|+\int_{\beta_{n}}^{x} \log x d F(x)<\frac{\varepsilon}{2} .
$$


Then

$$
\begin{aligned}
\mid \int_{1}^{x} \log x d F_{n}(x) & -\int_{1}^{\infty} \log x d F(x) \mid \\
& <\frac{\varepsilon}{2}+\left|\frac{1}{2^{n}} \sum_{i=1}^{2^{n-1}-1} \log \beta_{n, i}-\int_{\beta_{n, e}}^{\beta_{n}} \log x d F(x)\right| \\
& \leqslant \frac{\varepsilon}{2}+\frac{1}{2^{n}} \sum_{i=1}^{2^{n-1}-1} \log \left(\beta_{n, i} / \beta_{n, i+1}\right)
\end{aligned}
$$

as $F$ has weight $2^{-n}$ in each interval $\left(\beta_{n, i+1}, \beta_{n, i}\right)$, by Lemma 7

$$
=\frac{\varepsilon}{2}+2^{-n} \log \beta_{n}<\varepsilon
$$

for $n$ sufficiently large, using $5(\mathrm{c})$ again.

This lemma, combined with Lemma 4, proves Theorem 1.

\section{Proof of Theorem 2}

We now generalize the sequence $\left\{\beta_{n}\right\}$ by setting $\beta_{0}^{(b)}=b$, where $b$ is an odd positive integer, and $\beta_{n+1}^{(b)}>0$ by $H \beta_{n+1}^{(b)}=\beta_{n}^{(b)}(n=0,1, \ldots)$. By Lemma $4, \beta_{n}^{(b)}$ has degree $2^{n}$ over $Q$. Also, let $B_{n}^{(b)}$ be the generalisation of the set $B_{n}$, $B_{n}^{(b)}=\left\{\beta_{n}^{(b)}=\beta_{n, 1}^{(b)} \geqslant \beta_{n, 2}^{(b)} \geqslant \beta_{n, 3}^{(b)} \geqslant \ldots \geqslant \beta_{n, 2^{n}}^{(b)}\right\}$.

The next lemma allows us to approximate most elements of $B_{n}^{(b)}$ by elements of some $B_{j}$.

LEMMA 10. Apart from $\beta_{n}^{(b)}$ and $\left(\beta_{n}^{(b)}\right)^{-1}$, the other $2^{n}-2$ elements of $B_{n}^{(b)}$ can be arranged into disjoint pairs, so that there is a 1-1 correspondence between each pair $\beta_{n, i_{1}}^{(b)}, \beta_{n, i_{2}}^{(b)}$ and each element $\beta_{j, l}^{(1)}$ of $B_{0} \cup B_{1} \cup \ldots \cup B_{n-2}$, in such a way that $\left|\beta_{n, i_{1}}^{(b)}-\beta_{j . l}^{(1)}\right|$ and $\left|\beta_{n, i_{2}}^{(b)}-\beta_{j, l}^{(1)}\right|$ are less then $b^{-1}$.

Proof. The lemma is trivial for $n=1$. Assume it is true for $n$. For $B_{n+1}^{(b)}$, let the pair $H^{-1} \beta_{n, i_{1}}^{(b)}, H^{-1} \beta_{n, i_{2}}^{(b)}$ correspond to $H^{-1} \beta_{j, l}^{(1)}$. Then

$$
\left|H^{-1} \beta_{n, i_{1}}^{(b)}-H^{-1} \beta_{j,}^{(1)}\right|<\left|\beta_{n, i_{1}}^{(b)}-\beta_{j . l}^{(1)}\right|<b^{-1},
$$

by Lemma 5(a). This defines the correspondence for all elements of $B_{n+1}^{(b)}$ except $\left(H^{-1} \beta_{n}^{(b)}\right)^{ \pm 1}$ and $\left(H^{-1}\left(\beta_{n}^{(b)}\right)^{-1}\right)^{ \pm 1}$. The first two of these are $\left(\beta_{n+1}^{(b)}\right)^{ \pm 1}$, and so are excluded from the correspondence. Let the other two correspond to 1 . Then

$$
H^{-1}\left(\beta_{n}^{(b)}\right)^{-1}-1=H^{-1}\left(\beta_{n}^{(b)}\right)^{-1}-H^{-1} 0<\left(\beta_{n}^{(b)}\right)^{-1} \leqslant\left(\beta_{0}^{(b)}\right)^{-1}=b^{-1}
$$


by Lemma 5(a). Since $\left|x^{-1}-1\right|<|x-1|$ for $x>1$, the relevant inequality also holds for $\left(H^{-1}\left(\beta_{n}^{(b)}\right)^{-1}\right)^{-1}$. We have therefore obtained the required correspondence between $B_{n+1}^{(b)}$ and

$$
\begin{aligned}
\{1\} \cup H^{-1}\left(B_{0} \cup \ldots \cup B_{n-2}\right) & \cup\left(H^{-1}\left(B_{0} \cup B_{1} \cup \ldots \cup B_{n-2}\right)\right)^{-1} \\
& =B_{0} \cup B_{1} \cup \ldots \cup B_{n-1} .
\end{aligned}
$$

We can now prove Theorem 2.

Let $a>t$ and $a>0$ be given. We shall exhibit a $\beta_{n}^{(b)}$ with $\left|\log f_{n}^{(b)}-\log a\right|<\varepsilon$, where $f_{n}^{(b)}=\Omega\left(\beta_{n}^{(b)}\right)$. We first observe that a straightforward generalization of Lemma 5 (c) gives

$$
b \leqslant \beta_{n}^{(b)} \leqslant\left(2 n+b^{2}\right)^{\frac{1}{2}} .
$$

Also note that from Lemma 9(b) we may put

$$
\log \ell_{j}^{(1)}=\left(1-\delta_{j}\right) \log t
$$

where $\varepsilon_{j} \rightarrow 0$ as $j \rightarrow x$. Then by Lemma (10) and (5.1),

$$
\begin{aligned}
\log {\ell_{n}^{(b)}}_{n} & =\frac{1}{2^{n}} \sum_{i=1}^{2 n} \log \beta_{n, i}^{(h)} \\
& \geqslant \frac{1}{2^{n}}\left\{\log b+2 \sum_{j=0}^{n-2} \sum_{i=1}^{2 i} \log \left|\beta_{j, i}^{(1)}-b^{-1}\right|\right\} \\
& \geqslant 2^{-n} \log b+2 \sum_{j=0}^{n-2} 2^{-(n-j)} \log \ell_{j}^{(1)}+2 \sum_{j=0}^{n-2} 2^{-(n-j)} \log \left(1-b^{-1}\right) \\
& \geqslant 2^{-n} \log b+\left(1-2^{-(n-1)}\right) \log \ell-T_{n}-\log \left(1-b^{-1}\right)^{-1},
\end{aligned}
$$

where

$$
T_{n}=2 \sum_{j=0}^{n-2} 2^{-(n-j)}\left|\varepsilon_{j}\right| \log \ell
$$

Similarly, in the other direction

$$
\log \ell_{n}^{(b)} \leqslant 2^{-n} \log b+n 2^{-n} b^{-2}+\log \ell+T_{n}+\log \left(1+b^{-1}\right) .
$$

Now choose $N_{1}$ large enough so that

$$
112^{-n}+2^{-(n-1)} \log \ell+T_{n}<\frac{\varepsilon}{3} \text { for } n \geqslant N_{1} .
$$


We also want

$$
\left|2^{-n} \log b-\log (a / \ell)\right|<\frac{\delta}{3}
$$

or

$$
b \in\left(\left(\frac{a}{\ell} \exp \left(\frac{-\varepsilon}{3}\right)\right)^{2 n},\left(\frac{a}{\ell} \exp \left(\frac{\varepsilon}{3}\right)\right)^{2 n}\right) .
$$

Choose $N_{2} \geqslant N_{1}$ such that this interval contains an odd integer for $n \geqslant N_{2}$. Finally choose $N_{3} \geqslant N_{2}$ so that $\left.\max \left(\log \left(1-b^{-1}\right)^{-1}\right), \log \left(1+b^{-1}\right)\right)<\varepsilon / 3$ for $n \geqslant N_{3}$. The three $\varepsilon / 3$-inequalities now combine with (5.3) and (5.4) to give the required result.

\section{Small elements of $\mathscr{L}$}

We define a small element of $\mathscr{L}$ to be one in $[1, \ell]$. We now show that for $x_{q}$ defined by (1.3), $\Omega\left(\alpha_{q}\right)$ can only be small for finitely many $q$.

LeMma 11. We have

$$
\lim _{q \rightarrow x} \Omega\left(x_{q}\right)=\exp \frac{1}{2 \pi} \int_{0}^{2 \pi} \log _{+}\left|1-e^{i \theta}\right| d \theta=1.38135 \ldots
$$

ProOF. Now

$$
\begin{aligned}
\log \Omega\left(\alpha_{q}\right) & =\left(\frac{1}{2} \phi(q)\right)^{-1} \sum_{\substack{i=1 \\
(i . q)=1}}^{[q / 2]} \log _{+}|2 \cos (2 \pi i / q)| \\
& \rightarrow \frac{1}{2 \pi} \int_{0}^{2 \pi} \log _{+}\left|1-e^{i \theta}\right| d \theta \quad \text { ats } q \rightarrow \mathcal{J},
\end{aligned}
$$

since the discrepancy, on the unit circle, of the primitive $q$ th roots of 1 tends to 0 as $q \rightarrow x$. This fact follows, for instance, from Kuipers and Niederreiter (1974). Chapter 2, Theorem 2.5, and Hardy and Wright (1960). Theorem 272.

Now $\Omega\left(\alpha_{5}\right)=\Omega\left(\beta_{1}\right)$ is small, and $\Omega\left(\alpha_{7}\right)=1.309784 \ldots$ and $\Omega\left(x_{60}\right)=1.311254 \ldots$ are also small. We shall show, however, that these numbers also belong to a sequence of elements of $\mathscr{L}$ connected with fixed points of $H^{k}$ for some $k$. We need

LEMMA 12. For $k=1,2, \ldots, H^{k} x=P_{k}\left(x^{2}\right) / x Q_{k}\left(x^{2}\right)$, where $P_{1}(y)=y-1, Q_{1}(y)=1$ and

$$
P_{k+1}(y)=P_{k}^{2}(y)-y Q_{k}^{2}(y) \quad(k=1,2, \ldots)
$$




$$
Q_{k+1}(y)=P_{k}(y) Q_{k}(y)=\prod_{j=1}^{k} P_{k}(y),
$$

$$
P_{k}(y)=y^{.2-1}-\left(2^{k}-1\right) y^{2^{k-1}-1}+\ldots+1 \quad(k \geqslant 2) \text {, }
$$$$
Q_{k}(y)=y^{2^{k} 1-1}-\left(2^{k}-k-1\right) y^{2^{k}{ }^{1-2}}+\ldots-1 \quad(k \geqslant 2) \text {, }
$$

Further, $P_{k}$ is the minimal polynomial of $\beta_{k-1}^{2}$.

ProOF. Equation (6.1)-(6.6) all follow by induction, using the fact that

$$
H^{k+1} x=H\left(H^{k} x\right)=\frac{P_{k}\left(x^{2}\right)}{x Q_{k}\left(x^{2}\right)}-\frac{x Q_{k}\left(x^{2}\right)}{P_{k}\left(x^{2}\right)} .
$$

The final remark follows from the fact that $H \beta_{j}=\beta_{j-1}, H^{k} \beta_{k-1}=0$ and $H^{k}\left(-\beta_{k-1}\right)=0$.

Note that for $\varepsilon= \pm$, the roots of $H^{k} x=\varepsilon x$ are the zeros of $R_{\mu}^{\varepsilon}\left(x^{2}\right)$

We now establish a connection between the fixed points of $H^{k}$ and the values of $x$ where $F(x)$ is rational.

LEMma 13. (a) The values of $x$ where $F(x)=j /\left(2^{k}-1\right)\left(j=1,2, \ldots, 2^{k}-2\right)$ are the positive roots of $H^{k} x=x$ and of $H^{k} x^{-1}=x^{-1}$.

(b) The calues of $x$ where $F(x)=j /\left(2^{k}+1\right)\left(j=1,2, \ldots, 2^{k}\right)$ are the positive roots of $H^{k} x=-x$ and of $H^{k} x^{-1}=-x^{-1}$.

PrOOF. From (6.5), $H^{k} x=x$ and $H^{k} x^{-1}=x^{-1}$ each have $2^{k-1}-1$ positive roots, a total of $2 \mathrm{k}-2$. Let $F(x)=j / 2^{k}-1$, where $j \in\left\{1,2, \ldots, 2^{k}-2\right\}$. From (1.2), $F(\varepsilon H x)=\operatorname{res}(2 j \varepsilon) / 2^{k}-1$, where

$$
s=\left\{\begin{aligned}
1 & \text { if } x>1 \\
-1 & \text { if } x<1
\end{aligned}\right.
$$

and

$$
\operatorname{res}(a) \equiv u\left(\bmod 2^{k}-1\right), \quad \operatorname{res}(a) \in\left\{1,2, \ldots, 2^{k}-2\right\} .
$$

Hence, as $H\left(\varepsilon H^{i} x\right)=\varepsilon H^{i+1} x$, we can show by induction that for $\varepsilon^{\prime}=\operatorname{sgn}\left(H^{k} x-1\right)$,

$$
F\left(\varepsilon^{\prime} H^{k} x\right)=\frac{\operatorname{res}\left(2^{k} j \varepsilon^{\prime}\right)}{2^{k}-1} .
$$

Since res $\left(2^{k} j\right)=j, \operatorname{res}\left(-2^{k} j\right)=2^{k}-1-j$, and $\varepsilon^{\prime} H^{k} x=H^{k} x^{\varepsilon^{\prime}}, F\left(H^{k} x^{\varepsilon^{\prime}}\right)=F\left(x^{\varepsilon^{\prime}}\right)$.

Part (b) follows similarly. 
We now note that $H^{k} x= \pm x$ implies $H^{2 k} x=x$, and $H x=-x$ implies $H^{2 k+1} x=-x$. Hence, from Lemma $12, R_{2 k}^{+}(y)=2 k y^{2 k}+1-\ldots-1$ is divisible by $R_{k}^{+}(y) R_{k}^{-}(y)=2 k y^{2 k-1}-\ldots-1$, and $R_{1}^{-}(y)=2 y-1$ divides $R_{2 k+1}^{-}(y)=2 y^{2 * k}-\ldots+1$. Therefore, by defining

$$
S_{2 k}(y)=\frac{R_{2 k}^{+}(y)}{R_{k}^{+}(y) R_{k}^{-}(y)}, \quad S_{2 k+1}(y)=\frac{R_{2 k+1}^{-}(y)}{2 y-1},
$$

we obtain an infinite sequence of monic integral polynomials with constant term \pm 1 . Note that $S_{2 k}$ has degree $2^{2 k-1}-2^{k}$, and $S_{2 k+1}$ degree $2^{2 k}-1$. The $S_{i}$ need not be irreducible, as, for example, $S_{3} \mid S_{9}$. However, we can use the Möbius $\mu$-function to define, in a manner analogous to the formulae for irreducible cyclotomic polymials.

$$
S_{2 k}^{*}(y)=\prod_{k} S_{2 j}(y)^{\mu(k j)}, \quad S_{2 k+1}^{*}(y)=\prod_{j, 2 k+1} S_{j}(y)^{\mu(2 k+1) j)} .
$$

It is then possible that the $S_{i}^{*}$ may be irreducible. We have

$$
\begin{gathered}
S_{1}^{*}=S_{2}^{*}=1, \quad S_{3}^{*}(y)=y^{3}-5 y^{2}+6 y-1, \quad S_{4}^{*}(y)=y^{4}-7 y^{3}+14 y^{2}-8 y+1 . \\
S_{5}^{*}(y)=y^{15}-28 y^{14}+339 y^{13} \ldots-1 . \quad \text { etc. }
\end{gathered}
$$

It is easily checked that $S_{3}^{*}(y), S_{4}^{*}(y)$ are the minimal polynomials of $x_{7}^{2}, x_{60}^{2}$. Thus $x$-. $x_{60}$ arise naturally as roots of $H^{3} x=-x$, and $H^{4} x=x$, respectively.

Assuming that $S_{2 k}^{*}$ is irreducible, with $i_{2 k}$ a zero, then the absolute values of the conjugates of $; \gamma^{\frac{1}{2}} k$ are the values of $x$ where $F(x)=j\left(2^{2 h}-1\right)$, where

$$
2^{\frac{j}{k}-1} \neq \frac{j^{\prime}}{2^{k^{\prime}}-1} \text { for any } k^{\prime}<2 k
$$

Under the (likely) further assumption that these special values of $j\left(2^{2 h}-1\right)$ have small discrepancy in $[0,1]$, then

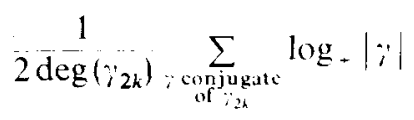

will be near

$$
\int_{1}^{x} \log x d F(x)
$$

i.e. $\Omega\left(i \frac{3}{2}\right)$ will be near $\ell$. This will be true whether $\operatorname{deg} ; i_{2 k}=2 \operatorname{deg} i_{2 k}$ or $\operatorname{deg} i_{2 k}$.

\section{References}

A. A. Albert (1965). Fundamental concepts of higher algebra (University of Chicago Press).

D. W. Boyd (1978). 'Variations on a theme of Kronecker'. Camod. Math. Bull. 2112 ). 129133. 
E. Dobrowolski (1979), 'On a question of Lehmer and the number of irreducible factors of a polynomial', Acta Arith. 34, 391.401.

G. M. Hardy and E. M. Wright (1960), An introduction to the theory of mumbers, 4th ed. (Oxford).

L. Kuipers and H. Niederreiter (1974). Uniform distribution of sequences (Wiley).

M. Mignotte (1978), 'Entiers algébriques dont les conjugués sont proches du cercle unité. Séminaire’ Delange-Pisot-Poitou (Thérie des nombres), 19e année, No. 39.

A. Schinzel (1973), 'On the product of the conjugates outside the unit circle of an algebraic number". Acta Arith. 24, 385399.

C. L. Stewart (1978), 'Algebraic incigers whose conjugates lie near the unit circle'. Bull. Soc. Math. France 106. $169-176$.

\section{Department of Mathematics}

James Cook University

Townsville, Queensland

Australia 4811 